\title{
Smart surgical template models for customized knee joint replace- ments
}

Philipp Sembdner, Technische Universität Dresden, Dresden, Germany, philipp.sembdner@tu-dresden.de Bernhard Bust, Technische Universität Dresden, Dresden, Germany, bernhard.bust@mailbox.tu-dresden.de Lars Dornheim, Dornheim Medical Images GmbH, Magdeburg, Germany, lars.dornheim@dornheim-medical-images.de Stefan Holtzhausen, Technische Universität Dresden, Dresden, Germany, stefan.holtzhausen@tu-dresden.de Ralph Stelzer, Technische Universität Dresden, Dresden, Germany, ralph.stelzer@tu-dresden.de

\section{Introduction}

Knee joint implants individually adapted to the patient are advantageous with regard to an exact fit in terms of size, shape and function. Appropriate software tools are necessary for digital planning and modeling based on the patient's computed tomography (CT) data to ensure optimal treatment of the patient. However, to take full advantage of customization, it is necessary to provide guidance to the clinician for accurate positioning of the implants during the procedure. It is therefore obvious to extend the process for developing patient-specific knee joint implants by automatically generating individual surgical templates followed by additive manufacturing.

\section{Methods}

To determine patient-specific parameters such as bone dimensions, an Active Shape Model (ASM) was developed that adapts to the bone topology and individualizes the parameters accordingly. The ASM model was trained with different training data sets. The data from diagnostic planning and ASM model are combined and applied to fully parametrically designed master models of necessary surgical templates in the computer aided design (CAD) system. In order to reduce the number of necessary templates, a categorization and evaluation depending on the surgical procedure, among others, was performed in the first step. As a result, nine templates were identified as necessary for a smooth surgical procedure.

\section{Results}

Features of common CAD systems like configurations, equations or the Application Programming Interface (API) were used during the development. Thus, the overall process and methodology of parametric model generation can be used in any CAD system. A developed software interface between the planning tool and the CAD system allows direct modification of the models without further forced intervention of the user. The fitting of the models was tested in specified ranges of values for each of the direct (from planning/ASM), dependent (calculation from direct dimensions), and fixed dimensions. The fitting worked without errors for the tested models. For selected datasets, the template models were transferred back to the planning tool for visual review on the patient CT dataset.

\section{Conclusion}

The smart model fitting of individual surgical templates offers potential for optimizing the previously time-consuming process chain of modeling. In combination with the automated generation of individual implant models and the use of novel additive manufacturing technologies, a fast and at the same time precise treatment of a patient is possible. In the further course of development, the adaptation of the ASM taking into account conspicuous ossifications (osteophytes) is planned for an even more precise and unambiguous application of the templates during surgery as well as the intuitive support of the surgeon through the automatic generation of suitable documentation (surgical procedure plan). 


\section{Current Development Overview of Torque-Limiting Smart Screw- driver for Orthopaedic Surgery}

Jack Wilkie, Institute of Technical Medicine, Hochschule Furtwangen, Villingen-Schwenningen, Germany, wij@,hsfurtwangen.de

Knut Moeller, Institute of Technical Medicine, Hochschule Furtwangen, Villingen-Schwenningen, Germany, moe@hsfurtwangen.de

\section{Introduction}

Bone screws are used in many orthopaedic procedures to stabilise fractures and fix implants. Correct torquing of these screws is important to prevent thread stripping or screw loosening and ensure the durability and longevity of these fixations. It has been proposed that a smart screwdriver may be able to monitor torque and rotation signals during screw insertion, and use model based methods to analyse the bone quality, and to determine the optimal insertion torque. In an ideal implementation, this would remove subjectivity from bone-screw tightening and may improve patient outcomes.

\section{Methods}

So far we have completed a variety of theoretical and validation studies towards the development of such a smartscrewdriver. Models of the bone-screw insertion have been formulated and tested for both identifiability with simulated data, and for accuracy using experimental data. Recently models have been formulated for estimating the optimal torque from the previously identified properties. A number of auxillary studies have attempted to quantify the effects of other factors on the accuracy of the system.

\section{Results}

We now have parts of the system to both determine the properties of bone from insertional data, and to estimate the torque from these. However there are still questions or problems related to quantifying and improving the accuracy and broad applicability of these methods, which must be addressed in future work.

\section{Conclusion}

From the currently completed research, we are well on the way towards proptotying a complete system, though a number of uncertainties remain, and there are additional factors to investigate. 


\section{Classification of scoliosis based on multiclass SVM model}

Animita Das, Department of EIE,RV College of Engineering, Bangalore,India, animitadas.lbi19@rvce.edu.in Dr.Tabitha Janumala,Department of EIE,RV College of Engineering, Bangalore,India,tabithajanumala@rvce.edu.in

\section{Introduction}

Scoliosis is defined as a sideways curvature of the vertebral column. About $40 \%$ to $80 \%$ of victims are adolescence. The current clinical approach uses radiographic images to determine the type of spine deformity and the severity of scoliosis is assessed by Cobb's Angle. There are different types of scoliosis based on the region of bend such as thoracic, thoracolumbar, lumbar, and double major. Classification of scoliosis is of utmost importance for guiding the treatment of scoliosis. This paper proposes an automated scoliosis classification system based on a multiclass SVM model that is trained with the extracted features from the scoliotic X-ray images using the pretrained deep neural networks to classify the scoliotic images into three categories Thoracic, Thoracolumbar, and Lumbar. A comparison was done using three pre-trained DNN.Statistical measures such as accuracy, Precision, Recall, and F1score were tabulated for each of them and overall accuracy of $50 \%$ was achieved

\section{Methods}

At the core of the work is a pretrained deep neural network (DNN), which is used as a feature extractor for the scoliotic $\mathrm{x}$ ray image dataset. This process, which transfer the knowledge of an existing DNN to a new domain, have been recently studied and proved successful for several applications. The main motivation of using this approach instead of fine-tuning the DNN is determined by several factors such as the size and the type of our dataset. Therefore, since our dataset is rather small the best option is to build a classier model on top of the output (activations) of the hidden layers. Furthermore, due to the mismatch between the dataset that was used to train the CNN and our dataset, is better to construct the classifier using the activations belonging to earlier layers of the network. The features extracted by the pretrained DNN are passed on to the multiclass SVM model for classification of the spine imageries.

\section{Results}

From the conducted study an overall accuracy of 50\% was achieved. Alex net and Squeeze Net showed higher accuracy for thoracic type of scoliosis and VGG-19 network showed higher accuracy for thoracolumbar type of scoliosis. Squeeze net showed higher accuracy and sensitivity for lumbar type of scoliosis

\section{Conclusion}

From the obtained results it can be concluded that pretrained DNN can be used as a feature extractor for efficient classification of scoliosis.However a better overall accuracy can be obtained by using a sufficiently larger dataset of spine imagery. 


\section{Assessing Generalisation Capabilities of CNN Models for Surgical Tool Classification}

Tamer Abdulbaki Alshirbaji, Institute of Technical Medicine (ITeM), Furtwangen University, Villingen-Schwenningen, Germany, and Innovation Centre Computer Assisted Surgery (ICCAS), University of Leipzig, Leipzig, Germany (e-mail: abd@hs-furtwangen.de).

Nour Aldeen Jalal, Institute of Technical Medicine (ITeM), Furtwangen University, Villingen-Schwenningen, Germany, and Innovation Centre Computer Assisted Surgery (ICCAS), University of Leipzig, Leipzig, Germany (e-mail: ja@hsfurtwangen.de).

Paul D. Docherty, Department of Mechanical Engineering, University of Canterbury, Christchurch, New Zealand, and Institute of Technical Medicine (ITeM), Furtwangen University, Villingen-Schwenningen, Germany (e-mail: paul.docherty@canterbury.ac.nz).

Thomas Neumuth, Innovation Centre Computer Assisted Surgery (ICCAS), University of Leipzig, Leipzig, Germany (email: thomas.neumuth@uni-leipzig.de).

Knut Moeller Institute of Technical Medicine (ITeM), Furtwangen University, Villingen-Schwenningen, Germany (email: moe@hs-furtwangen.de).

\section{Introduction}

Accurate recognition of surgical tools is a crucial component in the development of robust, context-aware systems. Recently, deep learning methods have been increasingly adopted to analyse laparoscopic videos. Existing work mainly leverages the ability of convolutional neural networks (CNNs) to model visual information of laparoscopic images. However, the performance was evaluated only on data belonging to the same dataset used for training. A more comprehensive evaluation of CNN performance on data from other datasets can provide a more rigorous assessment of the appraoches. In this work, we investigate the generalisation capability of different CNN architectures to classify surgical tools in laparoscopic images recorded at different institutions.

\section{Methods}

The generalisability of four CNN architectures of different depths and sizes was studied. The architectures were VGG16, ResNet-50, DenseNet-121 and EfficientNet-B0. In this study, two experiments were conducted using two datasets that contain laparoscopic videos of cholecystectomy procedures. One dataset was recorded at a single hospital (singlesite), whilst the other dataset was recorded at two sites (multi-site). The CNN models were pre-trained on ImageNet. In the first experiment, every pre-trained model was fine-tuned on the single-site dataset and evaluated on the multi-site dataset. In the second experiment, the pre-trained models were fine-tuned on the multi-site dataset and tested in the singlesite dataset.

\section{Results}

The mean average precision over the surgical tools observed in the first experiment reduced by $25 \%, 30 \%, 27 \%$, and $33 \%$ for VGG-16, ResNet-50, DenseNet-121 and EfficientNet-B0, respectively. Results of the second experiment showed better generalisation as the range of reduction in mean average precision was $8 \%$ to $15 \%$.

\section{Conclusion}

Experimental results imply that acceptable generalisation is possible, but only for surgical tools which are well presented in the training data. Nevertheless, this research highlight the need to determine the affect of using data from multiple sites on CNN generalisability. 


\section{Comparison of Geometrical Lung Models to Calculate Tidal Volumes during Spontaneous Breathing}

Simon Beck, IteM, Hochschule Furtwangen University, Villingen-Schwenningen, Germany, simon.beck@hs-furtwangen.de M.Sc. Bernhard Laufer, IteM, Hochschule Furtwangen University, Villingen-Schwenningen, Germany, b.laufer@hs-furtwangen.de Prof. Dr. Knut Möller, IteM, Hochschule Furtwangen University, Villingen-Schwenningen, Germany, moe@hs-furtwangen.de

\section{Introduction}

Measuring the tidal Volumes normally requires to connect a spirometer directly in the breathing flow of the patient. Due to medical conditions or environmental conditions in some cases this is uncomfortable, time consuming or even not possible. So in this research a motion capture system was used to collect data of the upper body to calculate the tidal breathing volumes.

\section{Methods}

Five People performed specific breathing patterns, varying in breathing rate and breathing depths. Shallow breathing $(\sim 200-500 \mathrm{ml})$, medium deep breathing $(\sim 2000-3000 \mathrm{ml})$ and deep breathing $(\sim 4000-6000 \mathrm{ml})$. XYZ-coordinates of 102 MoCap- markers, placed on a tight fit tshirt, surrounding the upper body, were collected with a Vicon ${ }^{\circledR}$ Motion tracking system and a Ganshorn Spirometer as a reference value.

Four lung models, containing 6 stacked geometric volumes of cylinders or truncated cones, based on a circle or an ellipse, were used to calculate the tidal volumes, using the Vicon ${ }^{\circledR}$ data.

\section{Results}

It was possible with each of the four models to calculate the breathing volumes during all three breathing depths. The mean root squared error werein each model were still significant $(50 \mathrm{ml}-400 \mathrm{ml})$, but the errors were higher with rising tidal volume.

The more anatomically fitting models based on cylinders and truncated cones using ellipses, as a base, showed better results than the ones based on circles of $13 \%$ on average.

\section{Conclusion}

The used models are a good method to estimate the breathing volume of people in a sitting position.

Further measurements should be done in the future to validate and improve the results of the used models and to test the method on people with different body shape, height and age.

It would also be interesting to see, if it possible to get reliably results when using a person in lying position.

To achieve this, the Numbers of MoCap-markers should also be reduced to make the measurement procedure faster and more practical.

Another use-case could be in analysis of medical fitness tests to get an estimation of the breathing volumina during exercise without the need for invasive measurements. 


\section{Changes of Physiological parameters of the patient during laparo- scopic gynecology}

Nour Aldeen Jalal, Institute of Technical Medicine (ITeM), Furtwangen University, Villingen-Schwenningen, Germany; and Innovation Center Computer Assisted Surgery (ICCAS), University of Leipzig, Leipzig, Germany, e-mail: nour.a.jala@hs-furtwangen.de

Tamer Abdulbaki Alshirbaji, Institute of Technical Medicine (ITeM), Furtwangen University, Villingen-Schwenningen, Germany; and Innovation Center Computer Assisted Surgery (ICCAS), University of Leipzig, Leipzig, Germany, e-mail: abd@hs-furtwangen.de

Paul D. Docherty, Department of Mechanical Engineering, University of Canterbury, Christchurch, New Zealand; and Institute of Technical Medicine (ITeM), Furtwangen University, Villingen-Schwenningen, Germany, paul.docherty@canterbury.ac.nz

Thomas Neumuth, Innovation Center Computer Assisted Surgery (ICCAS), University of Leipzig, Leipzig, Germany, thomas.neumuth@uni-leipzig.de

Knut Moeller, Institute of Technical Medicine (ITeM), Furtwangen University, Villingen-Schwenningen, Germany, moe@hs-furtwangen.de

\section{Introduction}

Analysing and fusing data from the medical devices of different discplines (anaesthesiology and surgery) inside the operating rooms (ORs) may promote awareness during surgical procedures. In this work, the changes of physiological parameters of patients undergoing laparoscopic procedures were analysed. This allowed statistical relationships between the intra-abdominal pressure and lung mechanics and hemodynamics changes to be investigated.

\section{Methods}

A dataset that contained synchronous real-time recordings from a patient monitor, anesthesia machine and surgical devices of seven patients undergoing gynaecological procedures was collected. The data were then screened to ensue completeness and correctness. The changes in the cardiovascular system, lung mechanics and intra-abdominal pressure during the procedure were evaluated. Moreover, information from the anesthesiological protocols were included. Patients were classified into Pressure controlled ventilation (PCV) and intermittent mandatory ventilation (IMV) groups.

\section{Results}

The results demonstrated that increasing the intra-abdominal pressure (IAP) resulted in increasing the airway peak pressure and decreasing the lung compliance. The Pearson correlation coefficient between the IAP and the airway peak pressure was 0.93 when changes of the ventilation settings were considered. Furthermore, different characteristic changes to the flow curve were observed when the PCV and the IMV modes were used. Major changes to the cardiovascular system included alterations in blood pressure and the oxygen saturation.

\section{Conclusion}

This work focused on studying the changes of physiological parameters of the patient during laparoscopic procedures and obtaining statistical relations between the intra-abdominal pressure, lung mechanics and cardiovascular changes. Future work will extend the concept of studying the relations between surgical actions and changes of vital signs of the patient. 